\title{
Portage Digestif des Bêta-Lactamases à Spectre Élargi Chez Quelques Patients de l'hôpital de la Caisse Nationale de Prévoyance Sociale de Maroua (Cameroun)
}

\author{
Maïworé Justine \\ Département des Sciences de la Vie et de la Terre, \\ Université de Maroua, Ecole Normale Supérieure, Maroua, Cameroun \\ Baane Martin-Paul \\ Laboratoire de l'hôpital de la caisse Nationale de Prévoyance Sociale, \\ Maroua, Cameroun \\ Youssouf Daouda \\ Bole Jean \\ Nango Nympeye Felicien Yvelin \\ Département des Sciences de la Vie et de la Terre, \\ Université de Maroua, Ecole Normale Supérieure, Maroua, Cameroun
}

Doi:10.19044/esj.2021.v17n10p107

Submitted: 10 July 2020

Accepted: 09 February 2021

Published: 31 March 2021
Copyright 2021 Author(s)

Under Creative Commons BY-NC-ND

4.0 OPEN ACCESS

Cite As:

Justine M., Martin-Paul B., Daouda Y., Jean B. \& Felicien Yvelin N.N. (2021). Portage Digestif des Bêta-Lactamases à Spectre Élargi Chez Quelques Patients de l'hôpital de la Caisse Nationale de Prévoyance Sociale de Maroua (Cameroun). European Scientific Journal, ESJ, 17(10), 107. https://doi.org/10.19044/esj.2021.v17n10p107

\section{Résumé}

La prise d'antibiotiques mal conservés et le non-respect de leur posologie et diverses contaminations peuvent être à l'origine de la résistance bactérienne. L'objectif de cette étude est d'évaluer le portage digestif des bêtalactamases à spectre élargi chez les patients de l'hôpital de la CNPS (Caisse Nationale de Prévoyance Sociale) de Maroua au Cameroun. Les bactéries du tube digestif ont été cultivées sur gélose Müller Hinton contenant des disques de céphalosporine de $3^{\text {ème }}$ génération (céftriaxone) et incubés à $37^{\circ} \mathrm{C}$ pendant 18 à 24 heures. Les bactéries résistantes ont ensuite été retenues, caractérisées et identifiées par utilisation des galeries API 20E. Les souches productrices de bêta-lactamases à spectre élargi ont ensuite été détectées par la technique de double synergie. Les résultats ont révélé que parmi les patients reçus, la 
tranche d'âge de 20-30 ans était la plus représentée et 93\% des patients avaient déjà pris des antibiotiques. Les bactéries telles que : Erwingella americana, Pantoea ssp, Serratia fumitica; Providenécia stuartii, Salmonella spp, Kluyvera spp, Cedecea spp, Klebsiella ozaenae, Vibrio cholerae et Pseudomonas spp ont été identifiées. Une forte résistance des souches identifiées a été observée vis-à-vis des antibiotiques suivants : l'amoxicilline $(88,22 \%)$, le céftriaxone $(88,22 \%)$, l'amoxicilline/acide clavulanique $(82,35 \%)$, le céftaxidime $(76,47 \%)$ et le céfotaxime $(70,59 \%)$. Dans l'ensemble, la prévalence des Bêta-lactamases à spectre élargi est très élevée parmi les malades fréquentant l'hôpital de la CNPS de Maroua (Cameroun).

Mots clés : Antibiotiques, $\beta$-lactamases à spectre élargi, Entérobactéries, Maroua (Cameroun), Portage digestif

\title{
Fecal Carriage of Extended Spectrum Beta-Lactamase Among Some Patients of the Hospital of the Caisse Nationale de Prévoyance Sociale in Maroua (Cameroon)
}

\author{
Maïworé Justine \\ Département des Sciences de la Vie et de la Terre, \\ Université de Maroua, Ecole Normale Supérieure, Maroua, Cameroun \\ Baane Martin-Paul \\ Laboratoire de l'hôpital de la caisse Nationale de Prévoyance Sociale, \\ Maroua, Cameroun \\ Youssouf Daouda \\ Bole Jean \\ Nango Nympeye Felicien Yvelin \\ Département des Sciences de la Vie et de la Terre, \\ Université de Maroua, Ecole Normale Supérieure, Maroua, Cameroun
}

\begin{abstract}
Taking poorly stored antibiotics and not respecting their dosage and various contaminants can cause bacterial resistance. The aim of this study was to evaluate the digestive carriage of beta-lactamases with an extended spectrum in patients attending the Hospital of the Medico-social Center of the CNPS (Casse Nationale de Prevoyance Sociale) in Maroua (Cameroon). Bacteria from the digestive tract were isolated on Müller Hinton agar containing 3rd generation cephalosporin (ceftriaxone) discs and incubated at $37^{\circ} \mathrm{C}$ for $18-24$ hours The resistant bacteria were then retained, characterized and identified using API 20E galleries. Broad spectrum beta-lactamase
\end{abstract}


producing strains were then detected using the double synergy technique. The results revealed that among the patients received, the 20-30 year age group was the most represented and $93 \%$ of the patients had already taken antibiotics. Among the microorganisms isolated in the inhibition zone, 23 strains were Gram negative and 35 Gram positive. Bacteria such as: Erwingella americana, Pantoea ssp, Serratia fumitica; Providencia stuartii, Salmonella spp, Kluyvera spp, Cedecea spp, Klebsiella ozaenae, Vibrio cholerae and Pseudomonas spp. have been identified. A Strong resistance of the identified strains was observed against the following antibiotics: amoxicillin (88.22\%), ceftriaxone (88.22\%), amoxicillin/clavulanic acid (82.35\%), ceftaxidime (76.47\%) and cefotaxime (70.59\%). Overall, the prevalence of extended spectrum beta-lactamases is very high among patients attending the CNPS hospital in Maroua (Cameroon).

Keywords: Antibiotics, Digestive carriage, Enterobacteriaceae, Extended spectrum $\beta$-lactamases, Maroua (Cameroon)

\section{Introduction}

Les infections bactériennes représentent $70 \%$ des cas de mortalités causées par les microorganismes (Walsh, 2003 ; Kemeze et al., 2016). Les maladies infectieuses représentent ainsi la seconde cause de décès et la première cause de perte de productivité au sein de la population active, à travers le monde (OMS 2006; Delmont et al., 2016). Les antibiotiques font partie des molécules les plus prescrites dans la lutte contre les bactéries pathogènes notamment en Afrique (Ouedraogo et al., 2017). Parmi ces antibiotiques, les bêta-lactamines représentent la famille la plus développée et la plus utilisée (Aminov, 2010 ; Challan, 2016). Cette forte utilisation des bêta-lactamines est due à leur large spectre, leur faible toxicité et leur efficacité (Gangoué, 2007). Pour ainsi poursuivre leur développement, les entérobactéries produisent deux (02) types de résistances face aux différents antibiotiques: une résistance naturelle et une résistance acquise. Les différentes formes de résistances bactériennes sont à l'origine de l'apparition des formes émergentes à l'instar des bactéries productrices de BLSE (bêtalactamase à spectre élargi) pour résister à la bêta-lactamine (Ouedraogo et al., 2017). Ceci représente le problème majeur rencontré dans la prise en charge thérapeutique (Ouedraogo et al., 2017).

$\mathrm{Au}$ cours des deux dernières décennies, les agents pathogènes résistants aux antibiotiques tels que certaines entérobactéries sont devenus un sérieux problème de santé publique. Une des raisons de l'augmentation de cette résistance pourrait résider dans l'utilisation préventive et thérapeutique d'antibiotiques chez les animaux dont les produits sont consommés par l'Homme (Corpet, 2000, Muller, 2017). 
A Maroua, la population consomme de la viande d'animaux issus des élevages dans lesquels, pour maintenir les bêtes en santé, l'usage des antibiotiques est courant. Les bactéries résistantes aux antibiotiques (bêtalactamases à spectre élargi) rencontrées dans les aliments et dans l'environnement peuvent entrer dans la chaine alimentaire et contaminer le tube digestif du consommateur (Maïworé et al., 2020 ; Lévi, 2006 ; Cantas et al., 2013). Un autre facteur d'apparition de résistance chez l'homme est lié aux antibiotiques eux-mêmes et non aux bactéries. La prise des antibiotiques mal conservés et le non-respect de leur posologie (dose et durée du traitement) peuvent être à l' origine de la résistance bactérienne. Ces phénomènes étant très observés dans notre localité, quelle peut être la prévalence du portage digestif des bêta-lactamases à spectre élargi chez les individus ? Pour évaluer cette prévalence des bêta-lactamases à spectre élargi chez ces populations, nous nous sommes fixés pour objectif de déterminer le portage digestif des bêta-lactamases à spectre élargi chez les patients fréquentant l'hôpital du Centre Médico-social de la CNPS de Maroua.

\section{Matériel et Méthodes}

\subsection{Site d'étude}

Cette étude a été réalisée dans la ville de Maroua, chef-lieu de la région de 1'Extrême-Nord Cameroun. Maroua est située entre $10^{\circ} 35^{\prime}-10^{\circ} 85^{\prime}$ ' latitude Nord et $14^{\circ} 14^{\prime}-14^{\circ} 19^{\prime}$ longitude Est avec une altitude moyenne de $423 \mathrm{~m}$. Cette ville abrite en son sein l'hôpital de la Caisse Nationale de Prévoyance Social (CNPS) qui représente notre site d'étude. La population de la ville de Maroua est estimée à 272.000 habitants qui représentent $10,6 \%$ de la population de l'Extrême-Nord et les 1,7 \% de celle du Cameroun.

\subsection{Matériel biologique}

Avant chaque prélèvement les informations telles que l'âge, le sexe et la fréquence d'utilisation des antibiotiques ont été recueillies. Le matériel biologique est constitué de 30 échantillons de selles provenant des patients venus de l'extérieur ou ceux hospitalisés (dès leur internement dans le service de Médecine générale). Le point commun entre les patients est qu'ils avaient des problèmes de santé et les examens de selles leur ont été prescrits. A l'aide d'un flacon-cuillère, l'équivalent d'une noix de selles fraîches émises par le patient a été transféré, dans un pot stérile. Ces différents flacons ont ensuite été transportés au laboratoire, étiquetés et conservés au frais dans un réfrigérateur puis analysées dans les deux heures qui suivent le prélèvement. 


\subsection{Préparation de l'inoculum et isolement des bactéries résistantes aux antibiotiques}

Avant l'isolement des bactéries, à l'aide d'une anse de platine, une pincée de selles fraîches a été prélevée puis écrasée contre la paroi du tube en verre contenant au préalable $3 \mathrm{~mL}$ d'eau distillée. Après homogénéisation pendant quelques secondes un boillon prêt à être ensemencé a été obtenu.

L'ensemencement s'est fait par écouvillonnage et a consisté à prélever une quantité de bouillon à l'aide d'un écouvillon et à l'étaler à la surface de la gélose Müller Hinton. Un disque de céphalosporine de $3^{\text {eme }}$ génération (céftriaxone) a été déposé sur la gélose et l'ensemble incubés à $37^{\circ} \mathrm{C}$ pendant 18 à 24 heures dans une étuve. Le disque de céftriaxone permet d'isoler les souches bactériennes résistantes ou intermédiaires à cet antibiotique. En effet l'antibiotique diffuse radialement dans le milieu et la croissance d'une bactérie s'arrêt dans la zone où la concentration d'antibiotique est supérieur à son seuil de résistance. Il se forme autour du disque un halo de diamètre plus ou moins grand. Cette zone peut contenir des tapis bactériens (résistants à l'antibiotique) ou non. Pour la suite de cette étude, les bactéries résistantes ont été retenues pour l'identification. La figure 1 ci-dessous nous permet d'observer une zone de halo d'inhibition.

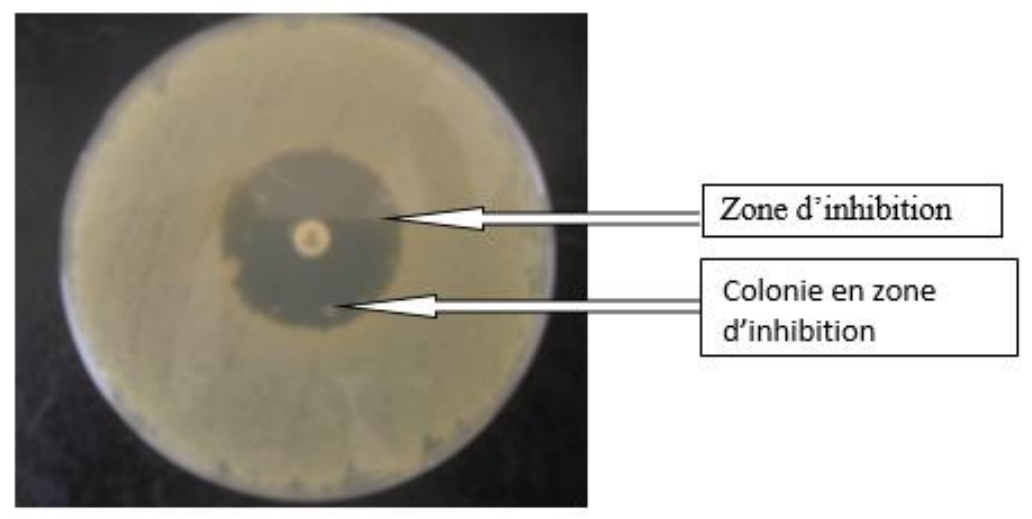

Figure 1 : Halo d'inhibition

\subsection{Caractérisation et identification des souches isolées}

Après incubation, des bactéries ont été observées. Autour des disques d'antibiotiques se sont formés des halos d'inhibition de diamètre plus ou moins grands. Cette zone peut contenir des tapis bactériens (résistants à l'antibiotique) ou non (Figure 1). Pour la suite du travail, seules les bactéries résistantes (celles présentes dans la zone d'inhibition) ont été retenues. La production des bêta-lactamases à spectre élargi est observée essentiellement chez les bactéries Gram négatif. Pour cette raison, seules ces dernières ont été retenues. Avant l'identification, les souches retenues ont tout d'abord été purifiées par des repiquages sur la gélose CLED (Cystine Lactose Électrolyte 
Déficient), milieu non sélectif permettant d'isoler les colonies bactériennes. Les bactéries ont ensuite été caractérisées en effectuant la coloration de Gram (Cheesbrough, 2006).

En vue de leur identification, les souches bactériennes sélectionnées ont été cultivées sur des galeries API 20E. Pour cela, un inoculum a été préparé : à l'aide d'une pipette pasteur, une colonie pure a été introduite dans un tube en verre contenant $3 \mathrm{ml}$ d'une solution physiologique $(\mathrm{NaCl} 8,5 \%$ ). Après homogénéisation la suspension bactérienne obtenue a été ensemencée sur les galeries API. Après incubation à $37^{\circ} \mathrm{C}$ pendant $24 \mathrm{~h}$, les résultats ont été observés. La lecture repose sur le changement de la coloration initiale des différents milieux : le test est positif lorsqu'il y'a virage de la coloration initiale. On obtient alors un code qui nous permet d'identifier la souche bactérienne en question par le biais de la base de donné Api 20E.

\subsection{Détection des BLSE}

2.5.1. Test de double synergie (Drieux et al., 2008)

Le test de double synergie a été utilisé pour déterminer les souches bactériennes productrices des BLSE. Ainsi, à l'aide d'une colonie prélevée sur la gélose C.L.E.D, un milieu Mac Farland 0,5 a été préparé et a servi à ensemencer le milieu Muller-Hinton. C'est sur ce milieu que sont déposés, à $30 \mathrm{~mm}$ de distance centre à centre, les disques d'antibiotiques. Autour d'un disque amoxicilline + acide clavulanique les disques de céftriaxone, céftazidime, céfotaxime et aztréoname ont été disposés comme décrit par Gangoué, 2007. En plus de ces disques, les disques d'imipenème et d'amoxicilline ont été ajoutés. Après une nuit dans une étuve, le résultat est décrété positif si on assiste à une augmentation de la zone d'inhibition autour du disque contenant la ceftriaxone, en direction du disque porteur d'acide clavulanique. En d'autres termes, c'est l'augmentation de la zone d'inhibition obtenue pour une céphalosporine en présence d'acide clavulanique, par rapport à la zone d'inhibition d'une céphalosporine seule, qui indique la présence d'une BLSE. Un test de synergie positif donne donc une image caractéristique, en «bouchon de Champagne» (effet potentialisateur de l'acide clavulanique) (Davison et al., 2000). La figure 2 présente la disposition des antibiotiques sur le milieu Muller Hinton.

Une fois les bêta-lactamases à spectre élargi detectées, l'interprétation de leur sensibilité vis-à-vis de l'antibiotique a été réalisée selon les critères du Comité de l'Antibiogramme de la Société Française de Microbiologie. Les différents résultats ont été classés en sensible (S), intermédiaire (I) et en résistant (R). Pour faciliter l'analyse des données les souches intermédiaires ont été considérées comme résistantes. 


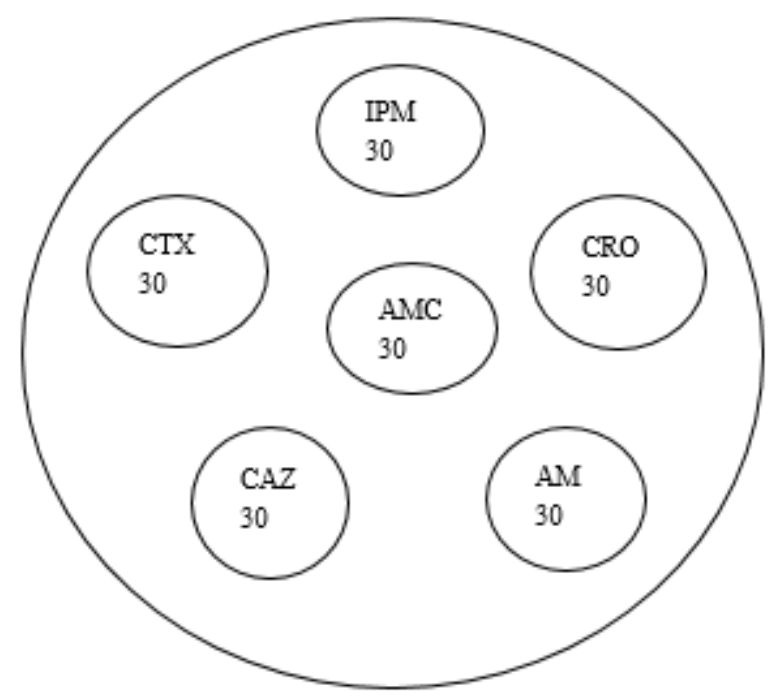

Figure 2 : Disposition des disques d'antibiotiques pour le test de double synergie. CTX : cefotaxime ; AMC : amoxicilline +acide clavulanique ; CAZ : céftazidime ; IPM : imipénème ; CRO : céftriaxone ; AM : amoxicilline ; 30 : charge de chaque antibiotique

\section{Resultats}

\subsection{Caractérisation des sujets ayant été impliqués dans l'étude}

3.1.1. Age, sexe et prise des antibiotiques chez les patients

\section{L'âge}

La caractérisation des populations étudiées a été faite sur la base de l'âge, du sexe et des antécédents de prise d'antibiotiques. La répartition en fonction de l'âge des individus étudiés, a permis d'obtenir le tableau 1. La tranche d'âge la plus touchée est celle de 20-30 avec 09 patients soit 30\% suivie de celle allant de $31-40$ avec 08 patients soit $26,66 \%$.

Tableau 1 : Représentation des patients en fonction de leurs âges

\begin{tabular}{ccc}
\hline Intervalle d'âge & Effectifs & Pourcentages \\
\hline $0-10$ ans & 07 & 23,33 \\
$11-20$ ans & 01 & 03,33 \\
$21-30$ ans & 09 & 30 \\
$31-40$ ans & 08 & 26,66 \\
$41-50$ ans & 04 & 13,33 \\
50 ans et plus & 01 & 03,33 \\
\hline TOTAL & 30 & 100 \\
\hline
\end{tabular}

\section{Le sexe}

Une répartition des patients en fonction du sexe a aussi été réalisée. Les résultats de cette analyse sont consignés dans le tableau 2. L'analyse de ce tableau révèle une prédominance du sexe féminin avec $63 \%$ contre $27 \%$ pour le sexe masculin soit un sex-ratio 1 homme pour 2,33 femmes. 
Tableau 2 : Représentation des patients en fonction du sexe

\begin{tabular}{ccc}
\hline Sexe de patients & Effectifs & Pourcentages \\
\hline Féminin & 19 & 63 \\
Masculin & 11 & 37 \\
\hline TOTAL & 30 & 100 \\
\hline
\end{tabular}

\section{Prise des antibiotiques}

Une répartition des patients en fonction de la prise antérieure d'antibiotiques a été réalisée et les résultats obtenus ont permis de répartir les malades en deux groupes. Un premier groupe constitué $93 \%$ d'individus et un second groupe constitué de $7 \%$ de patients dont l'âge est compris entre $0-10$ ans.

\subsection{Répartition des germes selon leurs caractères morphologiques}

Chez les 30 patients étudiés, au total 58 souches bactériennes ont été isolées. Elles sont constituées de 23 souches à Gram- et de 35 souches à Gram+ (Figure 3).

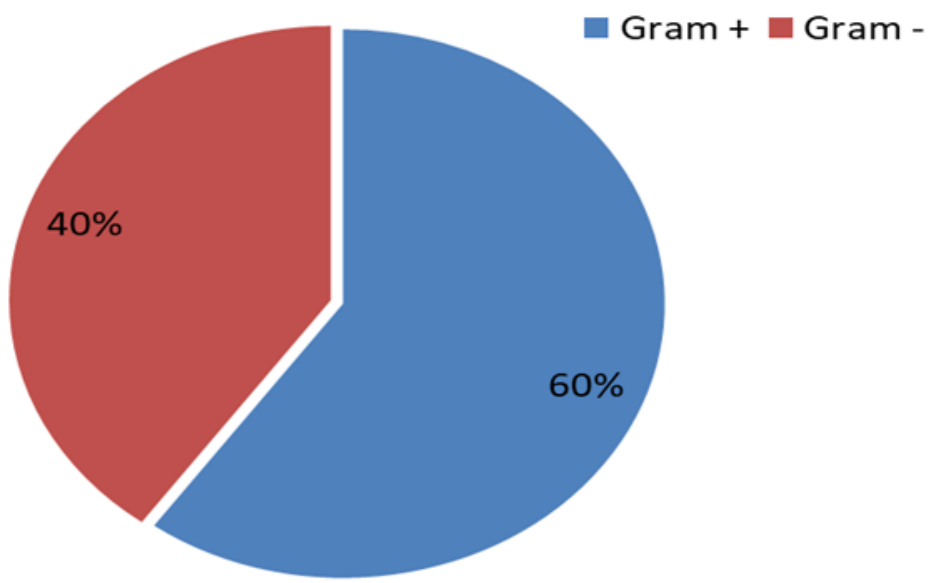

Figure 3 : Représentation des souches selon la coloration de Gram

Des 58 souches sensibles au céftriaxone isolées figurent 23 souches de bactéries à Gram- (soit 40\%) contre 35 souches à Gram+ (soit 60\%). Il y'a ainsi une forte représentation des souches à Gram+ qui n'ont présenté aucun intérêt pour cette étude. Les résultats obtenus ont également révélé que, parmi les 23 souches Gram négatif isolées, 17 entérobactéries ont été identifiées, avec un pourcentage de 73,91\% (Tableau 3), ce sont : Erwingella americana, Pantoea ssp, Serratia fumitica, Providencia stuartii, Salmonella spp, Kluyvera spp, Cedecea spp et Klebsiella ozaenae. L'analyse du tableau 3 révèle également la présence de 2 souches non-enterobactéries parmi lesquelles figurent : Vibrio cholerae et Pseudomonas spp. V. cholerae est le plus souvent responsable de toxi-infections alimentaires chez l'Homme. 
Tableau 3 : Répartition des entérobactéries et les non entérobactéries isolés

\begin{tabular}{lccc}
\hline Germes isolés & Effectifs & Pourcentage & Total des pourcentages \\
\hline Entérobactéries & & & $\mathbf{7 3 , 9 6}$ \\
Erwingella americana & 2 & 8,69 & \\
Pantoea ssp & 3 & 13,04 & \\
Serratia fumitica & 2 & 8,69 & \\
Providencia stuartii & 3 & 13,04 & \\
Salmonella spp & 2 & 8,69 & \\
Kluyvera spp & 2 & 8,69 & \\
Cedecea spp & 2 & 8,69 & $\mathbf{2 6 , 0 4}$ \\
Klebsiella ozaenae & 1 & 4,35 & \\
\hline Non Entérobactéries & & & \\
Vibrio cholerae & 3 & 13,04 & $\mathbf{1 0 0}$ \\
Pseudomonas spp & 3 & 13,04 & \\
\hline Total & 23 & 100 & \\
\hline
\end{tabular}

\subsection{Sensibilité aux antibiotiques}

La sensibilité des entérobactéries aux différents antibiotiques utilisés est représentée dans le Tableau 4. L'analyse de ce tableau révèle une forte résistance des souches à l'amoxicilline/acide clavulanique $(82,35 \%)$, au céftriaxone $(88,22 \%)$, au céftaxidime $(76,47 \%)$, au céfotaxime $(70,59 \%)$ et à l'amoxicilline $(88,22 \%)$. Seul l'Imipenème montre une forte sensibilité $(88,22 \%)$. Les résultats de la sensibilité ont montré que généralement les entérobactéries présentent un taux de résistance aux antibiotiques très élevé.

Tableau 4 : Sensibilité des entérobactéries aux différents antibiotiques utilisés

\begin{tabular}{lccccc}
\hline Nom de l'antibiotique & Valeurs critiques & Nombre de souches & \multicolumn{3}{c}{ Sensibilité (\%) } \\
\cline { 3 - 6 } & & & $\mathbf{R}$ & $\mathbf{I}$ & $\mathbf{S}$ \\
\hline Amoxicilline/Acide clavulanique & $14-21$ & 17 & 82,35 & 11,76 & 5,89 \\
Imipenème & $17-21$ & 17 & 5,89 & 5,89 & 88,22 \\
Céftazidime & $15-21$ & 17 & 76,47 & 17,64 & 5,89 \\
Céfotaxime & $15-21$ & 17 & 70,59 & 11,76 & 17,64 \\
Céftriaxone & $15-21$ & 17 & 88,22 & 11.78 & 00,00 \\
Amoxicilline & $14-21$ & 17 & 88,22 & 00,00 & 11,78 \\
\hline
\end{tabular}

$\mathbf{R}$ : résistant ; I : intermédiaire ; $\mathbf{S}$ : sensible

\subsection{Profil de résistance de différentes entérobactéries et Détection des Bêta-lactamases à spectre élargi.}

Pour établir le profil de résistance, les souches intermédiaires ont été considérées comme résistantes aux antibiotiques. Selon la sensibilité respective aux antibiotiques suivants Amoxicilline/Acide clavulanique, Imipenème, Céftazidime, Céftriaxone, et l'amoxicilline, différents profils de résistance ont été établis (Tableau 5). 
Tableau 5 : Profils de résistance des entérobactéries isolés.

\begin{tabular}{lccc}
\hline Profil de Résistance & $\begin{array}{c}\text { Nombre de } \\
\text { Colonies }\end{array}$ & $\begin{array}{c}\text { Colonies présentant } \\
\text { le profil }\end{array}$ & Pourcentage \\
\hline RSRRRR & 17 & 13 & 76,47 \\
RRRRSR & 17 & 2 & 11,76 \\
RSSRRR & 17 & 1 & 5,89 \\
RRRRRR & 17 & 1 & 5,89 \\
\hline
\end{tabular}

$\mathbf{R}$ : résistant ; S : sensible ; RSRRRR : sensible au $2^{\mathrm{e}}$ antibiotique et résistant aux autres ;

RRRRSR : sensible au $5^{\mathrm{e}}$ antibiotique et résistant aux autres ; RSSRRR : sensible aux 2é et $3^{\mathrm{e}}$ antibiotiques et résistant aux autres ; RRRRRR : résistant à tous les antibiotiques.

L'analyse du tableau 5 montre une prédominance du profil de résistance RSRRRR à $76,47 \%$ soit $13 / 17$ colonies. Ce profil de résistance correspond au profil de résistance des bêta-lactamases à spectre élargi qui est confirmés par le test de double synergie. La prévalence du phénotype BLSE chez les entérobactéries isolées est présentée dans le Tableau 6.

Tableau 6: Prévalence du phénotype BLSE chez les entérobactéries isolées

\begin{tabular}{lccc}
\hline Entérobactéries isolées & Nombre analysé & $\begin{array}{c}\text { Nombre présentant le } \\
\text { phénotype BLSE }\end{array}$ & Pourcentage \\
\hline Erwingella americana & 2 & 1 & 50,00 \\
Pantoea ssp & 3 & 2 & 66,66 \\
Serratia fumitica & 2 & 1 & 50,00 \\
Providencia stuartii & 3 & 2 & 66,66 \\
Salmonella spp & 2 & 2 & 100,00 \\
Kluyvera spp & 2 & 2 & 100,00 \\
Cedecea spp & 2 & 2 & 100,00 \\
Klebsiella ozaenae & 1 & 1 & 100,00 \\
\hline Total & $\mathbf{1 7}$ & $\mathbf{1 3}$ & $\mathbf{7 6 , 4 7}$ \\
\hline
\end{tabular}

En tenant compte de toutes les souches bactériennes isolées, la prévalence des Bêta-lactamases à spectre élargi est de 22,41\% (13/58); et lorsqu'on tient compte uniquement des entérobactéries elle est de 76,47\% (13/17).

\section{Discussion}

Au cours de cette étude, les tranches d'âge les plus concernées par la prise d'antibiotiques sont celles de 20 à 30 et 31 à 40 . Cette forte représentation de ces 2 tranches d'âge peut s'expliquer par une activité sexuelle élevée autour de ces âges car ces personnes généralement plus exposées à certaines infections sexuellement transmissibles ont recours aux antibiotiques par automédication ou par prescription pour se soigner. Cette forte représentation peut aussi s'expliquer par le fait que ces personnes font partie de la tranche active de la société, soucieuse de sa santé et ayant déjà une source de revenus. Ces résultats approchent ceux de Zomahou (2004) et de Podie (1999) qui ont trouvé que la tranche d'âge la plus représentée dans leurs travaux était respectivement celle de 25-39 ans et 20 à 50 ans à Cotonou. 
Parmi ces personnes les femmes sont plus représentées avec 63\%. Cette forte représentation du sexe féminin peut s'expliquer par la brièveté de l'urètre, la proximité entre l'orifice urinaire et l'orifice vaginal. En effet l'urine qui est le produit pathogène qui détient le plus de germes (Gangoué, 2007), peut permettre la transmission des germes vers l'orifice anal. En plus de ceci nous pouvons ajouter les grossesses, l'utilisation des gels spermicides et même des rapports sexuels qui peuvent contribuer à la dissémination de ces germes chez les femmes. Ces résultats concordent avec ceux de Podie (1999) réalisés à Cotonou au Bénin.

Parmi les personnes impliquées dans cette étude, 93\% avaient déjà pris des antibiotiques. Cette consommation excessive des antibiotiques par les patients peut s'expliquer par le large éventail de produits qu'offrent les antibiotiques, la facilité d'approvisionnement, la vente illicite au marché à moindre coût, l'utilisation des pénicillines en première intention dans les maladies infectieuses et surtout de l'émergence de la résistance bactérienne. Ces résultats confirment ceux de Sissoko (2000) à Bamako qui présente une utilisation des antibiotiques à $90 \%$ par les patients dans son étude.

Des 58 souches sensibles au céftriaxone isolées, les bactéries Gram + représentaient $60 \%$. Cette forte représentation se traduit par le fait que les bactéries Gram positif présentent une forte résistance aux béta-lactamines due à la structure de leur paroi. En effet en présence des béta-lactamines, ces bactéries ferment leurs pores pour empêcher leur entrée dans la cellule. Parmi les souches Gram négatif isolées qui représentaient 30\% des bactéries, 17 entérobactéries ont été identifiées. Leur présence s'explique par le fait que les entérobactéries sont des hôtes obligatoires du tube digestif de l'Homme et des animaux. Parmi les bactéries identifiées, figurent Vibrio cholerae et Pseudomonas spp., 2 souches non-enterobactéries. Les sérotypes de $V$. cholerae épidémiques pour l'Homme sont les sérotypes O1 et O139 sont majoritairement retrouvés dans les eaux stagnantes dans les pays en voie de développement (Helsens, 2018). Pseudomonas aeruginosa par contre a des sources de contamination humaine multiples, et peut être responsable d'infections graves. Cette bactérie présente une résistance naturelle à de nombreux traitements antibiotiques (Tzika, 2015).

Le test de résistance aux antibiotiques a révélé que la plupart des entérobactéries isolées présentent une forte résistance à tous les antibiotiques utilisés sauf à l'Imipenème avec un taux de résistance de 5,89\%. Ces résultats sont conformes à ceux obtenus par Gangoué (2007) à l'hôpital central de Yaoundé. Ce taux de sensibilité aux antibiotiques des entérobactéries est plus élevé que ceux généralement obtenus dans les pays en développement ceci peut s'expliquer par l'utilisation accrue et incontrôlées des bêta-lactamines dans ces pays. La détermination du profil de résistance a révélé une prédominance du profil RSRRRR avec une prévalence des Bêta-lactamases à 
spectre élargi élevée chez les entérobactéries isolées, soit 13 espèces sur les 17 identifiées. Ces résultats concordent avec ceux de Mirabaud (2003) à Genève sur les BLSE en pédiatrie qui a eu une prévalence des BLSE à 100\% avec le test de double synergie et à ceux de Ndiaye (2005) au CHN (Centre hospitalier National de Fann) qui a rapporté une prévalence des BLSE de 96\%. Cependant les résultats obtenus sont différents de ceux de Gangoué (2007) sur les BLSE à l'hôpital central de Yaoundé qui a rapporté une prévalence des BLSE de $12 \%$. Ces résultats confirment les différents résultats des études faites sur les BLSE dans les pays en développement à l'instar des travaux de (Xiong et al., 2002 ). La dissémination des BLSE observée s'explique par la forte utilisation des antibiotiques plus précisément de la classe des bêtalactamines (Kolar et al., 2001 ; Monnet et $a l ., 1998$ ). Ce qui est le cas de notre étude qui a montré une consommation des antibiotiques représentée à $93 \%$ (28 patients sur 30). Plusieurs autres facteurs comme l'automédication, l'utilisation progressive du test à double synergie au laboratoire peuvent aussi expliquer cette dissémination des BLSE (Nicolas-Chanoine, 2012).

\section{Conclusion}

Le présent travail avait pour but d'évaluer le portage digestif des bêtalactamases à spectre élargi chez les patients fréquentant l'hôpital du Centre Médico-social de la CNPS de Maroua. Chez les patients impliqués dans cette etude, la prise antérieure des antibiotiques était élevée, de 93\%. Ces souches isolées ont montré une forte résistance face aux antibiotiques utilisés, mis à part l'Imipenème qui a présenté une résistance de $5,89 \%$. Il en ressort au terme de cette étude une forte prévalence des Bêta-lactamases à spectre élargi observée chez les espèces suivantes : Erwingella americana, Pantoea ssp, Serratia fumitica, Providencia stuartii, Providencia stuartii, Salmonella spp , Kluyvera spp, Cedecea spp , Klebsiella ozaenae. Pour compléter cette étude, il faudra élargir le champ d'étude en tenant compte des autres produits pathogènes (urine, pus, sang et si possible le liquide céphalo-rachidien).

\section{References :}

1. Aminov, R.I. (2010). A brief history of the antibiotic era: lessons learned and challenges for the future. Front Microbiol., $1: 134$.

2. Cantas, L., Syed Q. A. Shah, L. M. Cavaco, C. M. Manaia, F. Walsh, M. Popowska, H. Garelick, Bürgmann, H., \& Sørum, H. (2016). A brief multi-disciplinary review on antimicrobial resistance in medicine and its linkage to the global environmental microbiota. Frontiers in Microbiology 4. doi: 10.3389/fmicb.2013.00096.

3. Challan, B. T. (2016). Les Bêta-lactamines. Service d'infectiologie Centre Hospitalier Alpes-Léman. 47 pages. www.infectiologie.com consulté le 3/07/2020. 
4. Cheesbrough, M. (2006) District Laboratory Practice in Tropical Countries, Part2 Second Edition, Cambridge, University Press, New York.; 190-250.

5. Corpet, D.E. (2000). Antibiotiques en agriculture et résistances bactériennes. In: Freney J, Renaud, F., Hanser, W., Bollet, C., eds., Précis de bactériologie clinique. Paris, Editions Eska.

6. Davison, H. C., Low, \& Woolhouse M. E. (2000). What is antibiotic resistance and how can we measure it? Trends Microbiol 8:554-9.

7. Delmont, J., Marchou B., Parola P., Pichard E. \& Simon F. (2016) ePILLY trop: Maladies infectieuses tropicales.@ CMIT et Alinéa 976 pages. www.infectiologie.com

8. Drieux, L., Brossier, F., Sougakoff, W., Jarlier, V. (2008). Phenotypic detection of extended-spectrum $\beta$-lactamase production in Enterobacteriaceae: review and bench guide. Clin Microbiol Infect 14:90-103.

9. Gangoué, J. P. (2007). Caracterisation des bêta-lactamases et leur inhibition par les extraits des plantes médicinales, thèse université de Liege.

10. Helsens, N. (2018). Étude de la résistance aux antibiotiques de souches de Vibrio parahaemolyticus isolées de produits de la pêche. Sciences du Vivant [q-bio]. dumas-02148585.

11. Kemeze, S., Moudze, B., Chiabi, A., Eposse, C., Kaya, A., Mbangue, M., Guifo, O. \& Kago, I. (2016). Profil clinique et bactériologique des infections néonatales bactériennes à l'Hôpital Laquintinie de Douala, Cameroun. Pan African Medical Journal. 23:97 doi:10.11604/pamj.2016.23.97.8523

12. Kolar, M., Ubranek., \& Lall, T. (2001). Antibiotic selective pressure and development of bacterial resistance. Inj antimicrob Agents.17:327-63.

13. Lévi, Y. (2006). Inquiétudes sur la présence d'antibiotiques et de bactéries antibiorésistantes dans les eaux. Environnement, Risques et Santé, 5: 261-265.

14. Maïworé, J. Youssouf., D., Toudjani, A. A., Baane, M-P., Tatsadjieu, Ngoune L. \& Montet D. (2020). Staphylococcus in Raw Cow's Milk from Maroua (Cameroon), Determination of their resistance level to antibiotics. Acta Scientific Microbiology 3. (4): 44-52.

15. Mirabaud I. M. (2003). Entérobactéries à bêta-lactamases à spectre élargi en pédiatrie en 1996. Thèse université de Genève $\mathrm{n}^{\mathrm{o}} 10303$.

16. Monnet, D.L., Archebal, L.K., Phillips, L., Tenover, F.C., McGowan, G.E. \& Gaynes L.P. (1998). Antimicrobial use and resistance in eight US hospitals: complexities of analysis and modeling. Infect control host Epidemiol. 19:388-394. 
17. Muller, A. (2017). Bon usage des antibiotiques: résultats d'actions dans différents types d'établissements de santé. Ecole doctorale environnement - sante, Université Bourgogne Franche-Comte, 194 p.

18. Ndiaye, A. O. (2006). Les Entérobactéries secrétrices de betalactamase à spectre élargie Thèse (Diplôme D'état), Université Cheikh Anta Diop De Dakar page:9.

19. Nicolas-Chanoine NH. (2012). Les entérobacteries productrices de bêta-lactamases à spectre élargi : où sont les dangers? Réanimation $2012 ; 21: 260-7$.

20. OMS (Organisation mondiale de la Santé) (2006). Bureau régional de l'Afrique. La santé des populations : le rapport sur la santé dans la Région africaine / Organisation mondiale de la Santé, Bureau régional de l'Afrique. ISBN 929031103192 pages

21. Ouedraogo A.S., Jean Pierre H., Banuls A.L., Ouedraogo R., Godreuil S. (2017).

22. Émergence et diffusion de la résistance aux antibiotiques en Afrique de l'Ouest : facteurs favorisants et évaluation de la menace. Medécine et Sante' Tropicales 2017, 27: 147-154

23. Podie, M. N. K. (1999). Evaluation de la sensibilité aux antibiotiques des germes les plus fréquemment isolés au laboratoire de bactériologie du CNHU de Cotonou (à propos de 896souches bactériennes isolées du 1 er Mars au 30 Juin 1999). Thèse de Med. N853, 145 pages.4557

24. Sissoko, R. (2000). Antibiothérapie dans le service de traumatologie de l'hopital Gabriel touré, Thèse, Pharm, Bamako, 00 p40.

25. Tzika, E., Ferrara, D., Boehncke, W.-H., Toutous Trellu L. \& Barouti, N. (2015) Surinfection de plaie chronique par Pseudomonas aeruginosa. Rev. Med. Suisse, $11: 768-772$.

26. Walsh, C. (2003). Antibiotics : actions, origins, resistances. Washington, D.C ASM press

27. Xiong, C., Zhu D., Wang., Zhang, Okamoto, Y.R \& Indoue A. (2002). Investigation of extended spectrum of Bêta- lactamases in kleibsiella Pneumoniae and Escherichia Coli from China. Diagn. Microbiol. infect. Dis 44:195-200.

28. Zomahoun, C. I. N. P. (2004). Evaluation de la sensibilité aux antibiotiques des bactéries isolées des infections urinaires au laboratoire de bactériologie du centre national hospitalier universitaire-Hubert Koutoukou Maga (C.N.H.U-H.K.M.) de Cotonou (A propos de 231 souches bactériennes isolées du 1er Avril au 31 Juillet 2004). Thèse, faculté de médecine de pharmacie et d'odontostomatologie, Mali. 107 pages. 